\title{
Study of InGaAs-based modulation doped field effect transistor structures using variable-angle spectroscopic ellipsometry
}

\author{
S. A. Alterovitz \\ National Aeronatics and Space Administration, Lewis Research Center, Cleveland, Ohio 44135 (U.S.A.) \\ R. M. Sieg* \\ Cleveland State University. Department of Electrical Engineering. Cleveland. Ohio 44115 (U.S.A.)
}

H. D. Yao, P. G. Snyder and J. A. Woollam

University of Nehraska. Department of Electrical Engineering. Lincoln. NE 68588 (U.S.A.)

J. Pamulapati and P. K. Bhattacharya

University of Michigan. Department of Electrical Engineering and Computer Science, Ann Arbor, MI 48109 (U.S.A.)

P. A. Sekula-Moise

Spire Corporation. Bedford. MA 01730 (U.S.A.)

\begin{abstract}
Variable-angle spectroscopic ellipsometry was used to estimate the thicknesses of all layers within the optical penetration depth of InGaAs-based modulation doped field effect transistor structures. Strained and unstrained InGaAs channels were made by molecular beam epitaxy (MBE) on InP substrates and by metal-organic chemical vapor deposition on GaAs substrates. In most cases. ellipsometrically determined thicknesses were within $10 \%$ of the growth-calibration results. The MBE-made InGaAs strained layers showed large strain effects, indicating a probable shift in the critical points of their dielectric function toward the InP lattice-matched concentration.
\end{abstract}

\section{Introduction}

$\operatorname{In}_{x} \mathrm{Ga}_{1-x}$ As can be grown epitaxially on InP or GaAs substrates, mostly as a strained layer. At a concentration of $x=0.53$, it is lattice matched to $\operatorname{InP}$. Although many applications in electronics and optoclectronics have been demonstrated using the latticematched concentration, superior properties of the strained-layer material have shifted the direction of applied research to $x \neq 0.53$. Modulation doped field effect transistors (MODFETs) with a strained-layer InGaAs channel have been demonstrated on InP [1] and on GaAs [2] substrates. Strain effects also play an important role in optoelectronic applications [3], especially near the band edge. Important parameters for any applications are the thicknesses of the layers, composition, interface quality and overlayer contamination or oxidation.

Ellipsometry, particularly variable-angle spectroscopic ellipsometry (VASE) in the visible, has been shown to characterize MODFETs [4] and optoelec-

*Undergraduate Student Intern at NASA Lewis Research Center. tronic structures [5] in the GaAs/AlGaAs family. This non-destructive optical technique can be used to perform a detailed analysis of all layers within the optical penetration depth. For InGaAs, this depth is of the order of $1000 \AA$, i.e. it covers all interesting layers in a MODFET structure. Ellipsometry has only been used twice in the past for InGaAs-based MODFETs $[6,7]$. The $\operatorname{In}_{x} \mathrm{Ga}_{1-x} \mathrm{As} / \mathrm{InP}$ MODFET with $x=0.53$ made by metal-organic chemical vapor deposition (MOCVD) was analyzed in detail, including interface quality, by Erman et al. [6]. However, the more common MODFET growth technique is molecular beam epitaxy (MBE) rather than MOCVD. In this technique, InP cannot be grown and is replaced by $\operatorname{In}_{0.52} \mathrm{Al}_{0.48} \mathrm{As}$, which is lattice matched to the InP substrate and provides the high band gap layer needed for the MODFET. Preliminary results on lattice-matched $\mathrm{In}_{x}$ $\mathrm{Ga}_{1-x}$ As/InAlAs/InP MODFETs were published by Alterovitz et al. [7]. However, no strain effects were reported in ref. 7. In this paper, we report on an ellipsometric study showing strain effects for the first time. The work included strained and lattice-matched InGaAs MODFETs made by MBE, as well as strained InGaAs MODFETs made by MOCVD on GaAs and 


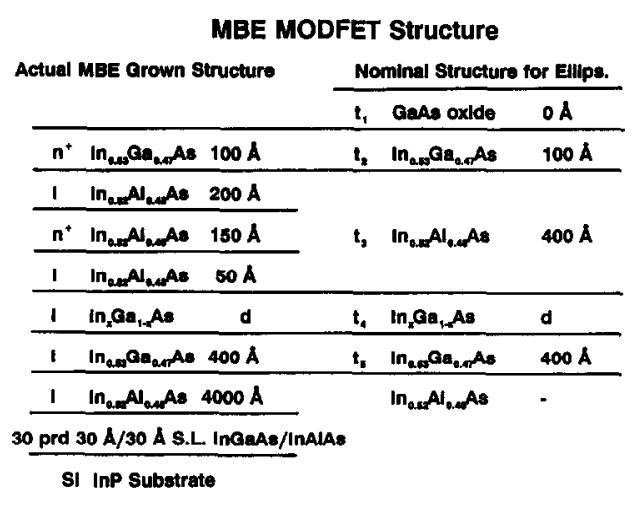

Fig. 1. Actual and nominal structures used in ellipsometry for the MBE-grown MODFET structures: $d=150 \AA$ for $x=0.53$ and 0.65 ; $d=100 \AA$ for $x=0.70$.

using AlGaAs as the donor layer. Results will be compared with growth-calibration data obtained during the crystal growth.

\section{Experimental details}

The structures grown on semi-insulating InP substrates were prepared by $\mathrm{MBE}$ at the University of Michigan. The structures are shown in Fig. 1. The 30 -period $30 \AA / 30 \AA$ superlattice buffer made of In$\mathrm{GaAs} / \mathrm{InAlAs}$ acts as a dislocation filter. The role of all the other layers is explained in ref. 7. Growth conditions are given in ref. 8. For ellipsometry purposes, the $4000 \AA$ thick InAlAs serves as the substrate, as the optical penetration depth in most of the experimental range is smaller than the thickness of all layers above the superlattice. Three indium concentrations $x$ were tested: $0.53,0.65$, and 0.70 . Nominal channel thicknesses derived from growth-calibration data are shown in Fig. 1.

Three samples grown by MOCVD at Spire Corporation on semi-insulating GaAs were also tested. The structures are shown in Fig. 2. The $1 \mu \mathrm{m}$ buffer is made mainly of iGaAs and is regarded as the substrate for ellipsometry purposes. Growth parameters are given in ref. 8 .

The ellipsometric technique used was similar to that described previously [9]. In this work, we minimized the mean square error $\sigma$ as defined in eqn. (1)

$$
\begin{aligned}
\sigma= & \left(N^{-1}\right) \sum_{i}\left[\left(\tan \psi_{\mathrm{e}, i}-\tan \psi_{\mathrm{c}, i}\right)^{2}\right. \\
& \left.+\left(\cos \Delta_{\mathrm{e}, i}-\cos \Delta_{\mathrm{c}, i}\right)^{2}\right]
\end{aligned}
$$

Here $\tan \psi_{\mathrm{e}, i}, \cos \Delta_{\mathrm{e}, i}$ are the experimental results and $\tan \psi_{\mathrm{c}, i}, \cos \Delta_{\mathrm{c}, i}$ are the model calculations. The summation is over all $N$ experimental points, i.e. all wavelengths and angles of incidence. Ellipsometric dielectric

\section{MOCVD MODFET STRUCTURE}

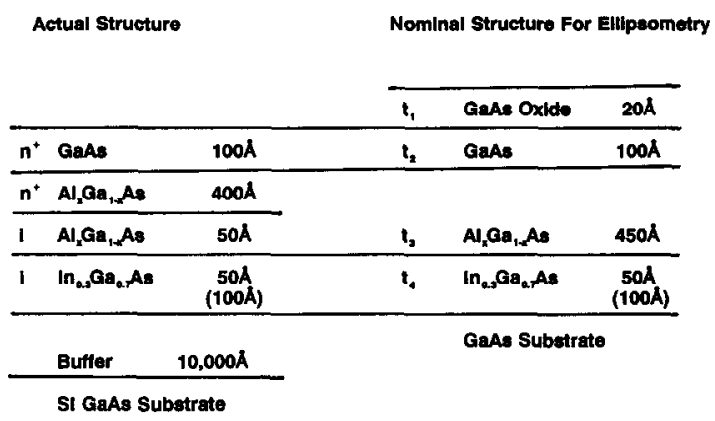

Fig. 2. Actual and nominal structures used in ellipsometry for the MOCVD-grown MODFET structures. The aluminum concentration in $\mathrm{Al}_{x} \mathrm{Ga}_{1-x} \mathrm{As}$ is a variable. Some samples have a $50 \AA$ $\mathrm{In}_{0.3} \mathrm{Ga}_{0.7} \mathrm{As}$ layer and others have a $100 \AA$ layer.

calibration functions were taken from the following: $\mathrm{In}_{x} \mathrm{Ga}_{1}{ }_{x}$ As from the algorithm developed in ref. [8], $\mathrm{AlGaAs}$ from the algorithm given in ref. 10, GaAs from ref. $11, \mathrm{In}_{0.52} \mathrm{Al}_{0.48} \mathrm{As}$ from previous measurements at NASA Lewis Research Center and the oxide from ref. 12. The dielectric function for the oxide was measured on GaAs oxides, but it gives a good description of the oxide on $\mathrm{In}_{0.53} \mathrm{Ga}_{0.47}$ As [13].

\section{Results and discussion}

Results for three indium compositions grown by MBE are given in Table 1 . The thicknesses $t_{i}$ correspond to Fig. 1. The quality of the fits, as measured by the value of the mean square error, is good. However, two features of these results were unexpected: the very low values of the cap InGaAs layer thicknesses and the negative values for the strained layer thicknesses. The low values obtained for the cap layers can be explained by the very short growth time of these layers and the accompanying experimental errors, and by possible loss of material due to oxidation. We found this result for the InGaAs cap layer in all our MBE samples. Another explanation might involve an interface layer between the InAlAs and the cap layer. However, for our structures it is almost impossible to study this interface by ellipsometry. We already have 4-5 parameters and an interface layer would introduce another parameter. In addition, we already have a high correlation parameter ( 0.9 or higher) between $t_{2}$ and $t_{3}$, the cap layer and the InAlAs layer thicknesses respectively. The correlation problem was discussed in refs. 14 and 15 . The high correlation between $t_{2}$ and $t_{3}$ means that ellipsometry has difficulty in estimating accurately the value of each one of these two parameters, but the sum $t_{2}+t_{3}$ is not affected by the correlation. Results given in Table 1 support this conclusion, with experimental $t_{2}+t_{3}$ values 
TABLE 1. Best fits for MBE-grown InGaAs/InAlAs MODFET structures using the strain-free calibration functions (wavelength range $3300-7500 \AA)$

\begin{tabular}{|c|c|c|c|c|c|c|}
\hline & \multicolumn{2}{|l|}{$x=0.53$} & \multicolumn{2}{|l|}{$x=0.65$} & \multicolumn{2}{|l|}{$x=0.70$} \\
\hline & Nominal & Ellipsometry & Nominal & Ellipsometry & Nominal & Ellipsometry \\
\hline$t_{1}$ & 0 & 20.8 & 0 & 27.6 & 0 & 25.8 \\
\hline$t_{2}$ & 100 & 45.7 & 100 & 41.4 & 100 & 50.3 \\
\hline$t_{3}$ & 400 & 443 & 400 & 452 & 400 & 424 \\
\hline$t_{4}$ & 150 & & 150 & -0.5 & 100 & -28.8 \\
\hline$t_{5}$ & 400 & 553 & 400 & 566 & 400 & 559 \\
\hline$\sigma$ & - & $3.2 \times 10^{-3}$ & --- & $2.7 \times 10^{3}$ & & $1.8 \times 10^{-3}$ \\
\hline
\end{tabular}

TABLE 2. Best fits for MBE-grown strained-layer channel samples, assuming all InGaAs layers are lattice matched to InP, i.c. $x=0.53$ (wavelength range $33007500 \AA$ )

\begin{tabular}{|c|c|c|c|}
\hline \multicolumn{2}{|l|}{$x=0.65$} & \multicolumn{2}{|l|}{$x=0.70$} \\
\hline Nominal & Ellipsometry & Nominal & Ellipsometry \\
\hline 0 & 27.5 & 0 & 26.7 \\
\hline 100 & 41.5 & 100 & 48.9 \\
\hline 400 & 452 & 400 & 433 \\
\hline 150 & & 100 & \\
\hline 400 & 564 & 400 & 530 \\
\hline 0.65 & 0.53 & 0.70 & 0.53 \\
\hline.- & $2.6 \times 10^{-3}$ & & $1.8 \times 10^{-3}$ \\
\hline
\end{tabular}

in the range $474-493 \AA$, while the nominal value is $500 \AA$.

The other unexpected result, the negative values for $t_{4}$, is definitely related to the special property of this layer, namely that it is a pseudomorphic strained layer. The ellipsometric dielectric calibration functions that were used for the calculations were taken from ref. 8, where a study of strain-free $\mathrm{In}_{x} \mathrm{Ga}_{1-x}$ As material was done. It is known that the band gap of pseudomorphic InGaAs grown on InP is different from that of unstrained bulk InGaAs [16]. There are no theoretical calculations of the dielectric function of pseudomorphic
InGaAs or InP in the visible. However, we expect the critical points and, accordingly, the dielectric function [10] to change continuously as a function of composition. In the case of pseudomorphic InGaAs, we find a split of the valence band [16] and the resulting band structure with the associated critical points is expected to be more complex than the case for AlGaAs. Ilowever, in a very rough approximation and extension of the result in ref. 16, the band gap of strained-layer $\mathrm{In}_{x} \mathrm{Ga}_{1-}$ As is similar to unstrained $\operatorname{In}_{y} \mathrm{Ga}_{1,}$ As, where $x>y$ for $y>0.53$, i.e. for compressive strain. Assuming that all critical points move in the same direction we have to use as a calibration dielectric function for our strained $\operatorname{In}_{x} \mathrm{Ga}_{1-x}$ As the dielectric function obtained for unstrained $\mathrm{In}_{y} \mathrm{Ga}_{1}$ "As from ref. 8 with $x>y$. We used the dielectric function of the unstrained $\operatorname{In}_{0.53} \mathrm{Ga}_{0.47} \mathrm{As}$ to fit the $\mathrm{In}_{x} \mathrm{Ga}_{1,} \mathrm{As}$ strained-lattice layers for both $x=0.65$ and 0.70 . The results are given in Table 2 . The quality of the fits is good, and there are no unphysical results for layer thicknesses. Results are also shown in Fig. 3(a) and (b) for the fit given in Table 2 for the $x=0.70$ sample. There is a remarkably good fit of the experimental and the modeled data, including the sharp jumps in the values of $\cos \Lambda$.

Two comments on the results given in Table 2 will now be discussed. First comment is with regard to

TABLE 3. Best fits for MOCVD-grown InGaAs/AlGaAs MODFET structures using the strain-free calibration functions (wavelength range $3500-6800 \AA$ A)

\begin{tabular}{|c|c|c|c|c|c|c|}
\hline & \multicolumn{2}{|c|}{ MO6-316-3 } & \multicolumn{2}{|c|}{ MO6-332-1 } & \multicolumn{2}{|c|}{ MO6-334-1 } \\
\hline & Nominal & Best fit & Nominal & Best fit & Nominal & Best fit \\
\hline$t_{1}$ & 20 & 20.6 & 20 & 17.7 & 20 & 18.3 \\
\hline$I_{2}$ & 300 & 341 & 100 & 101 & 100 & 103 \\
\hline$x$ & 0.2 & 0.223 & 0.2 & 0.166 & 0.2 & 0.155 \\
\hline$t_{3}$ & 450 & 374 & 450 & 443 & 450 & 491 \\
\hline$t_{4}$ & 100 & 86.0 & 100 & 94.5 & 50 & 52.8 \\
\hline$\sigma$ & ... & $3.3 \times 10^{-4}$ & - & $5.7 \times 10^{-4}$ & - & $5.0 \times 10^{-4}$ \\
\hline
\end{tabular}




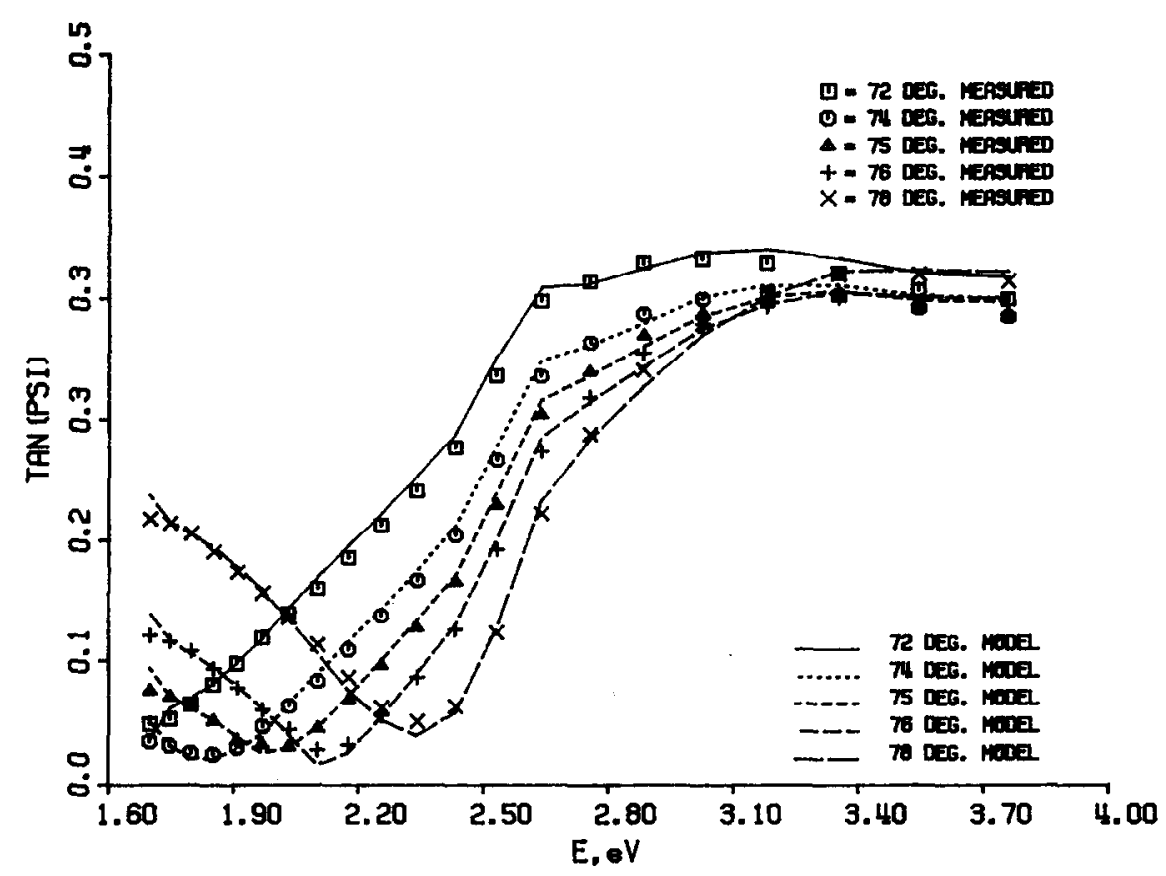

(a)

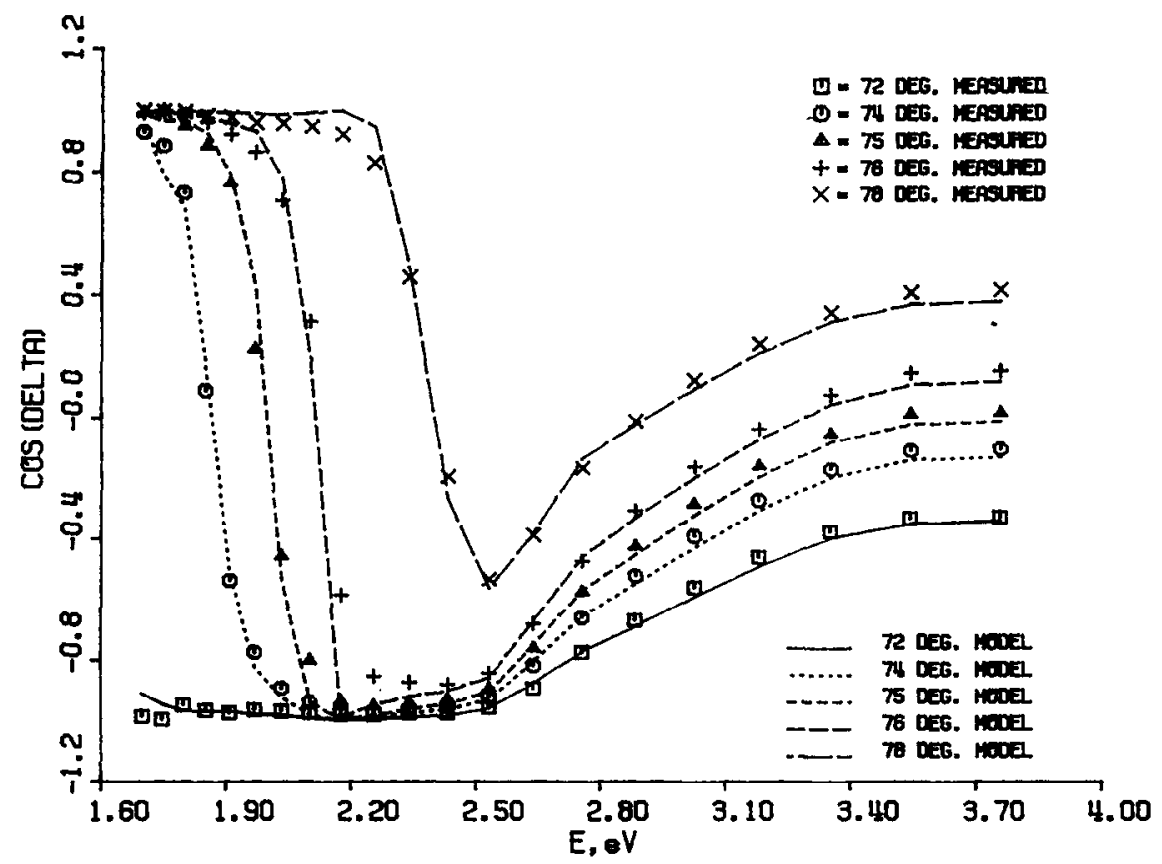

(b)

Fig. 3. Experimental and model simulation for (a) $\tan \psi$ and (b) $\cos \wedge$ v. energy for five angles of incidence for the MBE-grown $x=0.70$ sample. All of the InGaAs material is assumed to be lattice matched to InP.

correlations. The same correlation problem between $t_{2}$ and $t_{3}$ mentioned previously was also observed for the results given in Table 2 . We will now combine the results of Table 1 (for the lattice-matched sample) and Table 2 (for the strained-lattice sample) for the parameter $t_{2}+t_{3}$ : while the nominal value is $500 \AA$ for all samples, the range of experimental results is 482 $493 \AA$, even smaller than the range mentioned above. The second comment is with regard to the concentration parameter $x$, especially for the strained-lattice samples. This parameter was obtained from growthcalibration data, which were cross checked by X-rays 


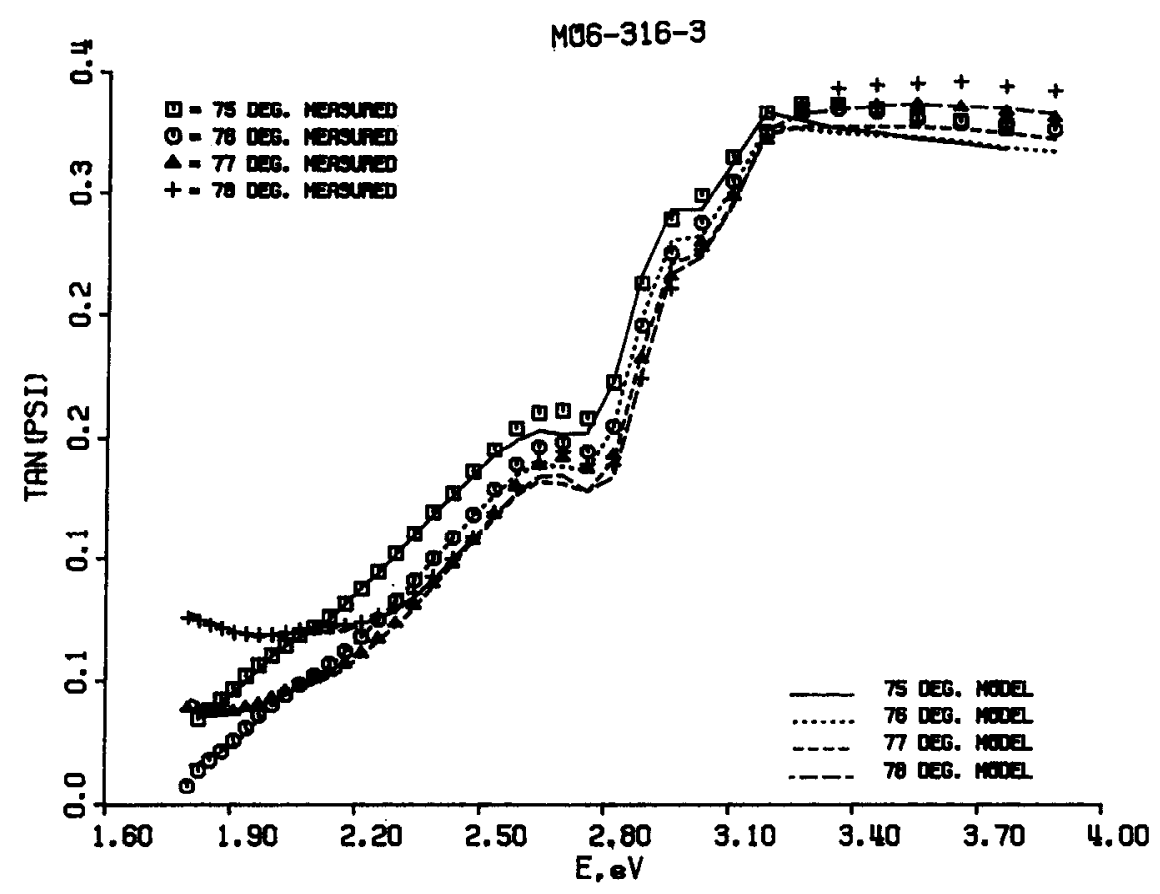

(a)

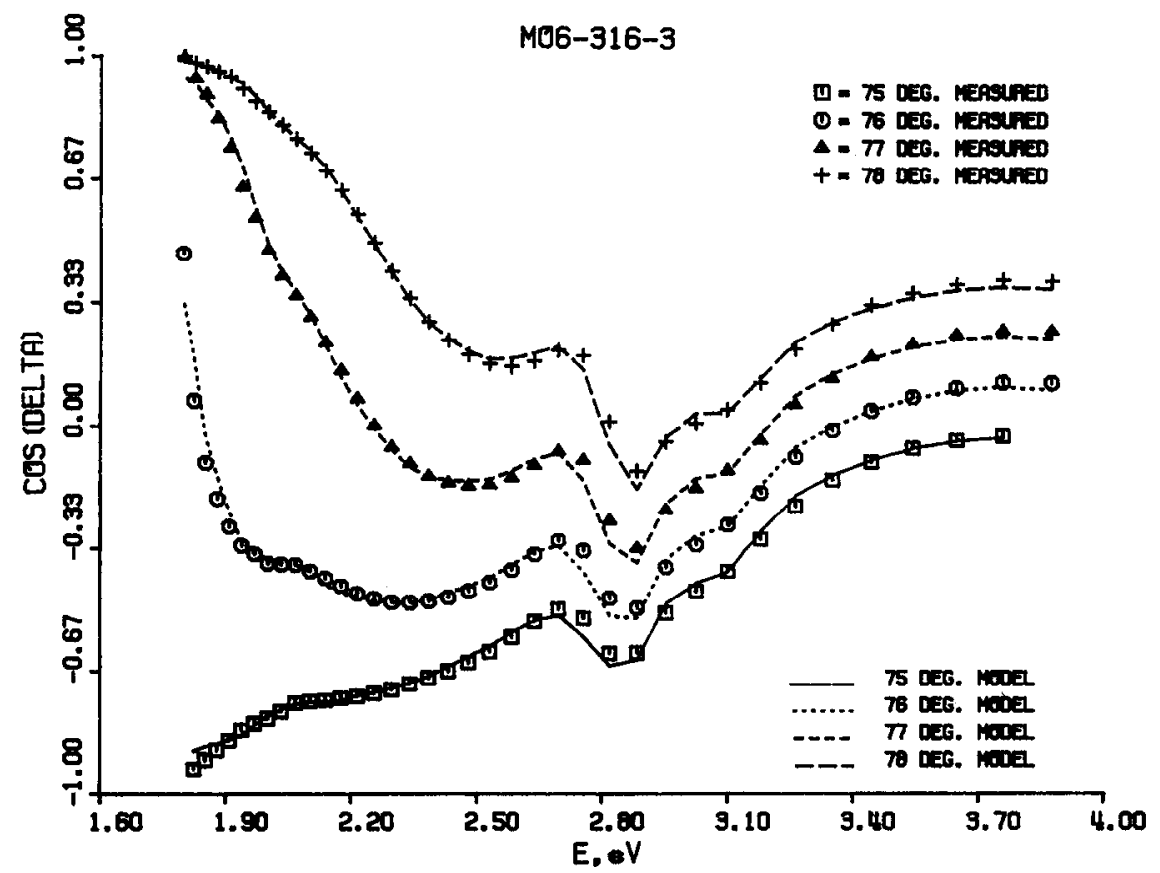

(b)

Fig. 4. Experimental and model simulation for (a) $\tan \psi$ and (b) $\cos \Delta v$ s. energy for four angles of incidence for an MOCVD-grown $x=0.30$ sample.

for strain-free material. A positive confirmation that $x$ for the strained-lattice samples is not $53 \%$ was obtained from electrical measurements of devices made from the same wafers or similarly prepared wafers $[17,18]$. The measured device parameters show a clear dependence on the nominal value of $x$.
Three MOCVD-grown MODFET structures of pseudomorphic $\mathrm{In}_{0.3} \mathrm{Ga}_{0.7}$ As on $\mathrm{GaAs}$ were measured. The results are shown in Table 3. For one of the samples, we also show a complete comparison of experiment and model in Fig. 4. The mean square errors $\sigma$ are much smaller than those for the InGaAs/InAlAs/InP 
samples. In addition, all results pertaining to the In$\mathrm{GaAs}$ and the GaAs layer thicknesses are in good agreement with the nominal values. There are some discrepancies between the ellipsometric estimates and the AlGaAs nominal concentrations or thicknesses. At the present time, we believe that the ellipsometric result may be a more accurate description of the sample. The low values for $\sigma$ are probably due to better calibration spectra for the constituents and the fact that the aluminum concentration in $\mathrm{AlGaAs}$ is a free parameter, while the composition in $\operatorname{In}_{0.52} \mathrm{Al}_{0.48}$ As is held constant.

\section{Conclusions}

A variety of InGaAs MODFET structures, made by both MBE and MOCVD were studied by variableangle spectroscopic ellipsometry. The thicknesses of all layers involved in the electric conduction were estimated and reasonably good fits to the nominal values were obtained. We have clearly seen, for the first time, strain effects in pseudomorphic InGaAs. These effects were explained qualitatively as a shift of the critical points in the strained $v s$. the unstrained InGaAs material. In addition, in the case of MOCVD, we have seen possible problems in an AlGaAs layer, showing that ellipsometry can be used as a quality control tool for a complex material.

\section{Acknowledgment}

We would like to thank Mark Drotos for technical help in the ellipsometric analysis.

\section{References}

1 G. I. Ng, W. P. Hong, D. Pavlidis, M. Tutt and P. K. Bhattacharya, IEEE Electron Devices Lett., 9 (1988) 439.

2 W. T. Masselnik, J. Klem, T. Henderson, A. Ketterson, J. S. Gedjmin, H. Morkoc and K. R. Gleason, Proc. Int. Electron Devices Meet. (IEDM) 1985, p. 755.

3 S. C. Hong, G. P. Kothigal, N. Debbar, P. K. Bhattacharya and J. Singh, Phys. Rev. B, 37 (1988) 878.

4 P. G. Snyder, M. C. Rost, G. H. Bu-Abbud, J. A. Woollam and S. A. Alterovitz, J. Appl. Phys., 60 (1986) 3293.

5 S. A. Alterovitz, J. A. Woollam and P. G. Snyder, Solid State Technol., 31 (1988) 99.

6 M. Erman, J. P. Andre and J. LeBris, J. Appl. Phys., 59 (1986) 2019.

7 S. A. Alterovitz, R. M. Sieg, H. D. Yao, P. G. Snyder, J. A. Woollam, J. Pamulapati and P. K. Bhattacharya, Mater. Res. Soc. EA-2I (1990) 299.

8 S. A. Alterovitz, R. M. Sieg, H. D. Yao, P. G. Snyder, J. A. Woollam, J. Pamulapati, P. K. Bhattacharya and P. A. SekulaMoise, Proc. ICEM-90, in the press.

9 S. A. Alterovitz, R. M. Sieg, N. S. Shoemaker and J. J. Pouch, Mater. Res. Soc. Symp. Proc., 152 (1989) 21

10 P. G. Snyder, J. A. Woollam, S. A. Alterovitz and B. Johs, J. Appl. Phys., 68 (1990) 5925.

11 D. E. Aspnes and A. A. Studna, Phys. Rev. B, 27 (1983) 985.

12 D. E. Aspnes, G. P. Schwartz. G. J. Gualtieri, A. A. Studna and B. Schwartz, J. Electrochem. Soc., 128 (1981) 590.

13 D. E. Aspnes and H. J. Stocker, J. Vac. Sci. Technol., 21 (1982) 413.

14 S. A. Alterovitz. G. H. Bu-Abbud, J. A. Woollam and D. C. Liu, J. Appl. Phys., 54 (1983) 1559.

15 G. H. Bu-Abbud, N. M. Bashara and J. A. Woollam, Thin Solid Films, 138 (1986) 27

16 R. People, Appl. Phys. Lett., 50 (1987) 1604.

17 R. Lai, P. K. Bhattacharya, S. A. Alterovitz, A. N. Downey and C. Chorey, Electron Devices Lett., 11 (1990) 564.

18 J. Pamulapati, R. Lai, G. I. Ng, Y. C. Chen, P. R. Berger, P. K. Bhattacharya, J. Singh and D. Pavlidis, J. Appl. Phys.; 68 (1990) 347. 